\title{
hnRNP A1 and A/B Interaction with PABPN1 in Oculopharyngeal Muscular Dystrophy
}

\author{
Xueping Fan, Christiane Messaed, Patrick Dion, Janet Laganiere, \\ Bernard Brais, George Karpati and Guy A. Rouleau
}

\begin{abstract}
Background: Oculopharyngeal muscular dystrophy (OPMD) is an adult-onset disorder characterized by progressive ptosis, dysphagia and proximal limb weakness. The autosomal dominant form of this disease is caused by short expansions of a (GCG) 6 repeat to $(\mathrm{GCG})_{8-13}$ in the PABPN1 gene. The mutations lead to the expansion of a polyalanine stretch from 10 to $12-17$ alanines in the N-terminus of PABPN1. The mutated PABPN1 (mPABPN1) induces the formation of intranuclear filamentous inclusions that sequester poly(A) RNA and are associated with cell death. Methods: Human fetal brain cDNA library was used to look for PABPN1 binding proteins using yeast two-hybrid screen. The protein interaction was confirmed by GST pull-down and co-immunoprecipitation assays. Oculopharyngeal muscular dystrophy cellular model and OPMD patient muscle tissue were used to check whether the PABPN1 binding proteins were involved in the formation of OPMD intranuclear inclusions. Results: We identify two PABPN1 interacting proteins, hnRNP A1 and hnRNP A/B. When co-expressed with mPABPN1 in COS-7 cells, predominantly nuclear protein hnRNP A1 and A/B colocalize with mPABPN1 in the insoluble intranuclear aggregates. Patient studies showed that hnRNP A1 is sequestered in OPMD nuclear inclusions. Conclusions: The hnRNP proteins are involved in mRNA processing and mRNA nucleocytoplasmic export, sequestering of hnRNPs in OPMD intranuclear aggregates supports the view that OPMD intranuclear inclusions are "poly(A) RNA traps", which would interfere with RNA export, and cause muscle cell death.
\end{abstract}

RÉSUMÉ: Interaction de hnRNP A1 et A/B avec PABPN1 dans la dystrophie musculaire oculopharyngée. Introduction: La dystrophie musculaire oculopharyngée (DMOP) est une maladie de l'âge adulte caractérisée par une ptose progressive des paupières, une dysphagie et une faiblesse musculaire proximale. La forme autosomique dominante est causée par de courtes expansions d'une répétition (GCG)6 à (GCG)8-13 dans le gène PABPN1. Les mutations donnent lieu à une expansion d'un tractus de polyalanine de 10 à 12-17 alanines dans la partie N-terminale de PABPN1. Le gène PABPN1 muté (PABPN1m) induit la formation d'inclusions filamenteuses intranucléaires qui séquestrent l'ARN poly(A) et entraînent la mort cellulaire. Méthodes: Une librairie d'ADNc provenant de cerveau foetal humain a été utilisée pour chercher la protéine liant PABPN1 au moyen du système à double-hybrides dans la levure. L'interaction protéine-protéine a été confirmée par GST pull-down et co-immunoprécipitation. Le modèle cellulaire de DMOP et le tissu musculaire provenant de patients atteints DMOP ont été utilisés pour vérifier si les protéines liant PABPN1 étaient impliquées dans la formation des inclusions intranucléaires dans la DMOP. Résultats: Nous avons identifié deux protéines interagissant avec PABPN1, hnRNP A1 et hnRNP A/B. En co-expression avec PABPN1m dans des cellules COS-7, les protéines hnRNP A1 et A/B à prédominance nucléaire se retrouvent avec PABPN1m dans les agrégats intranucléaires insolubles. Des études chez les patients atteints de DMOP ont montré que hnRNP A1 est séquestré dans les inclusions nucléaires. Conclusions: Les protéines hnRNP sont impliquées dans la maturation de l'ARNm et le transport nucléocytoplasmique de l'ARNm. La séquestration de hnRNPs dans les agrégats intranucléaires appuie l'hypothèse selon laquelle les inclusions intranucléaires de la DMOP sont des "piètes à ARN poly(A)" qui interfèrent avec le transport de l'ARN et causent la mort des cellules musculaires.

Can. J. Neurol. Sci. 2003; 30: 244-251

Autosomal dominant oculopharyngeal muscular dystrophy (OPMD) is an adult-onset disease that presents in the fifth or sixth decade. The disease is characterized by progressive eyelid drooping (ptosis), swallowing difficulties (dysphagia) and proximal limb weakness. ${ }^{1-6}$ Pathological studies showed the presence of unique intranuclear filamentous inclusions in skeletal muscle fibers of OPMD patients. ${ }^{7,8}$ The OPMD locus was mapped by linkage analysis to chromosome $14 \mathrm{q} 11.1^{9-11}$ and the gene was identified as PABPN1, encoding the poly(A) binding protein nuclear 1 (PABPN1, PABP2, PAB II). ${ }^{12}$ Dominant OPMD is caused by expansion of a short GCG trinucleotide repeat in the PABPN1 gene. The normal PABPN1 gene has a $(\mathrm{GCG})_{6}$ trinucleotide repeat coding for a polyalanine stretch at the 5' end, while in OPMD patients this (GCG) ${ }_{6}$ repeat is expanded to $(\mathrm{GCG})_{8-13}$. Due to the presence of GCA GCA

From the Center for Research in Neuroscience, McGill University, and the McGill University Health Center, (XF, CM, PD, JL, GAR); †Centre de recherche du CHUM, Hôpital Notre-Dame, Université de Montréal, (BB); Montreal Neurological Institute, McGill University (GK), Montreal, Quebec, Canada.

ReCEIVED OCtoBer 2, 2002. ACCEPTED IN FINAL FORM MARCH 5, 2003.

Reprint requests to: Guy A. Rouleau, Rm L7-224, Montreal General Hospital, 1650 Cedar Avenue, Montreal, Quebec, Canada. 
GCA GCG coding sequences adjacent to the (GCG) 6 repeat, the wild-type PABPN1 has a 10 alanine stretch in its $\mathrm{N}$-terminus, while the mutant proteins have 12 to 17 alanines.

In addition to OPMD, at least five other diseases are associated with alanine stretch expansions in the disease gene products. Synpolydactily is caused by an alanine stretch expansion from 15 to $22-25$ in the HOXD13 gene, ${ }^{13}$ while cleidocranial dysplasia is associated with an expansion from 17 to 27 alanines in CBFA1 protein. ${ }^{14}$ The alanine stretch lengthening from 15 to 25 in ZIC2 protein results in holoprosencephaly (HPE), ${ }^{15}$ whereas the ones expanded from 15 to 22-33 in HOXA13 protein and from 14 to 24 in FOXL2 protein cause hand-foot-genital syndrome ${ }^{16}$ and type II blepharophimosis/ ptosis/epicanthus ${ }^{17}$ respectively. Among these alanine-expanded proteins, mutated PABPN1 (mPABPN1) is the only one that is reported to induce the formation of intranuclear inclusions. Oculopharyngeal muscular dystrophy intranuclear inclusions are similar to those found in a number of inherited neurodegenerative diseases caused by mutated proteins with an expanded polyglutamine (polyQ) stretch encoded by a CAG repeat. The mutant form of each protein typically has a polyQ tract of greater than 40 glutamine residues, whereas the wild-type protein may contain around 20 glutamines. ${ }^{18}$ This group of disorders includes Huntington's disease, ${ }^{19}$ spinobulbar muscular atrophy, ${ }^{20}$ dentatorubral pallidoluysian atrophy, ${ }^{21,22}$ and the spinocerebellar ataxias type 1, 2, 3, 6 and 7.23-29

PABPN1 has 306 amino acids and is comprised of an alanine stretch and a proline-rich region in the N-terminus, a ribonucleoprotein (RNP)-type RNA binding domain in the central region, and an arginine-rich C-terminus. PABPN1 is an abundant nuclear protein that binds with high affinity to the poly(A) tail of mRNA and is involved in mRNA polyadenylation, ${ }^{30}$ a two-step reaction whereby endonucleolytic cleavage of the nascent mRNA transcript is followed by an addition of $\sim 250$ adenylate residues to the up-stream cleavage product. ${ }^{31-33}$ Poly(A) tail synthesis is catalyzed by poly(A) polymerase through interaction with CPSF, the cleavage and polyadenylation specificity factor. However, this process is slow and inefficient, and the length of poly(A) tail is poorly controlled. Adding PABPN1 to this reaction will stimulate poly(A) synthesis and control the size of the tail to be $\sim 250 \mathrm{nt}$ in length. ${ }^{30,34,35}$

PABPN1 has been identified as a component of the filamentous inclusions present in the nuclei of OPMD muscle fibers. ${ }^{36-38}$ Expression of mPABPN1 in COS-7 cells induces the formation of intranuclear protein aggregation that is associated with cell death. ${ }^{39-42}$ PABPN1 contains two oligomerization domains that make the protein form oligomers. ${ }^{40}$ Oligomerization of mPABPN1 facilitates the formation of OPMD nuclear inclusions. ${ }^{40}$ These inclusions contain ubiquitin, the subunits of the proteasome, poly(A) RNA, ${ }^{36}$ and PABPN1 interacting protein SKIP. ${ }^{41}$ A recent study showed that overexpression of chaperone proteins can reduce aggregation of mPABPN1 and cell death. ${ }^{42}$ To further investigate how OPMD intranuclear protein aggregates cause cell death, we searched for PABPN1 interacting proteins using yeast two hybrid library screen and found that both heterogeneous nuclear ribonucleoprotein (hnRNP) A1 and A/B interact with the Cterminus of PABPN1. These hnRNP proteins are sequestered in the OPMD nuclear inclusions. The hnRNPs are mRNA binding proteins and involved in mRNA export from the nucleus to cytoplasm. ${ }^{43}$ The fact that OPMD intranuclear aggregates sequester hnRNP proteins supports the hypothesis that the OPMD aggregates impair mRNA nucleocytoplasmic export and cause cell death.

\section{MeTHODS}

\section{Yeast two-hybrid screening and cloning of hnRNP A1 and} A/B.

The DupLEX-A ${ }^{\mathrm{TM}}$ yeast two-hybrid system (OriGene Tech, Rockville, MD) was used to identify PABPN1 interacting proteins. To generate bait for the yeast two-hybrid screening, the cDNA encoding the C-terminus of PABPN1 from amino acids 249 to 306 was cloned with EcoR1/BamH1 into vector pEG202NLS that allows the fusion of the bait to the DNA binding domain of LexA. A human fetal brain cDNA library (OriGene Tech, Rockville, MD) was constructed in vector pJG45 that fuses proteins to the transcription activation domain of B42. The lacZ gene in construct pSH18-34 and LEU2 gene in the genome of yeast strain EGY48 were used as reporter genes. The pEG202NLS-AA249-306, pSH18-34 and library construct pJG4-5 were co-transformed into EGY48. The clones were characterized as positive if the yeast cells turn blue in the presence of X-gal and grow in the absence of leucine. EST clones 549503 and 610850 encoding the full-length cDNA of hnRNP A1 and hnRNP A/B, respectively, were obtained from IMAGE.

The full-length human PABPN1 cDNA and the cDNA encoding AA287-306 of PABPN1 were cloned into vector pEG202 with EcoR1/BamH1. The full-length cDNAs of hnRNP $\mathrm{A} 1$ and A/B were cloned into vector pJG4-5 with EcoR1/Xho1. All constructs were verified by DNA sequencing. $\beta$ galactosidase liquid assays was performed exactly as described. ${ }^{40}$ The $\beta$-galactosidase unit is calculated using the formula $\left[\mathrm{A}_{420} \times 1000\right] / \mathrm{A}_{600} \times$ time (in min) $\mathrm{x}$ volume (in ml)].

\section{In vitro binding assay}

To make GST-PABPN1 fusion protein, the coding cDNA of wtPABPN1 was cloned into the GST vector pGEX-5X-1 (Amersham Pharmacia Biotech, Piscataway, NJ) allowing the fusion of GST to the N-terminus of PABPN1. The construct was verified by DNA sequencing and transformed into E. Coli XL1Blue. The Bulk and RediPack GST purification modules (Amersham Pharmacia Biotech, Piscataway, NJ) were used for expression and purification of GST-PABPN1 according to manufacturer's instructions. Purified proteins were analyzed by Western blotting using anti-GST antibody. The cDNAs of hnRNP A1 and A/B were cloned with EcoR1/Xho1 into the vector pJG4-6 that allows the fusion of the HA tag to the Ntermini of $\mathrm{A} 1$ and $\mathrm{A} / \mathrm{B}$. HA tagged hnRNP A1 and A/B were expressed in the yeast strain EGY48 and confirmed by Western blotting using the antibody against the HA epitope. GST pulldown assay was performed exactly as described..$^{40}$

\section{Immunofluorescence}

The cDNAs encoding the wild-type PABPN1 (wtPABPN1) and polyalanine-expanded PABPN1 with 17 alanines 
(mPABPN1) were cloned with EcoR1/BamH1 into the GFP (green fluorescent protein) vector pEGFP-C2 (Clontech, Palo Alto, CA) which allows the fusion of GFP to the N-terminus of PABPN1 proteins. The cDNAs of hnRNP A1 and A/B were cloned into pEGFP-C2 (with the GFP gene removed) using EcoR1-Xho1. The HA tag sequence was inserted into the construct at the EcoR1 site. The constructs were verified by DNA sequencing. COS-7 cells were transfected or co-transfected using Lipofectamine reagent (Gibco BRL, MD) according to the manufacturer's instructions. In 72 hours, the transfected cells were fixed with $4 \%$ paraformaldehyde and immunodetection was performed using an anti-HA antibody (Santa Cruz Biotech, CA) (1:500). Rhodamine conjugated secondary antibody (1:500) was used, and the signals were visualized using a fluorescent microscope. To remove the soluble proteins, the co-transfected cells were treated with $0.5 \mathrm{M}$ potassium chloride $(\mathrm{KCl})$ in HPEM (30 mM HEPES, 65 mM Pipes, $10 \mathrm{mM}$ EDTA, $2 \mathrm{mM} \mathrm{MgCl}$, $\mathrm{pH}$ 6.9) for five minutes at room temperature. Then the cells were fixed and immunodetected using an anti-HA antibody.

The microtome sections of paraffin-embedded deltoid muscle from an OPMD patient and a control subject were used. Sections were deparaffinized, permeabilized and immunostained with polyclonal anti-PABPN1 antibody or monoclonal antibody against hnRNP A1. Rhodamine conjugated secondary antibody was used and the signal was visualized using a fluorescent microscope. To remove the soluble proteins, the deparaffinized sections were treated with $1 \mathrm{M} \mathrm{KCl}$ in HPEM for five minutes at room temperature before the immunostaining was performed.

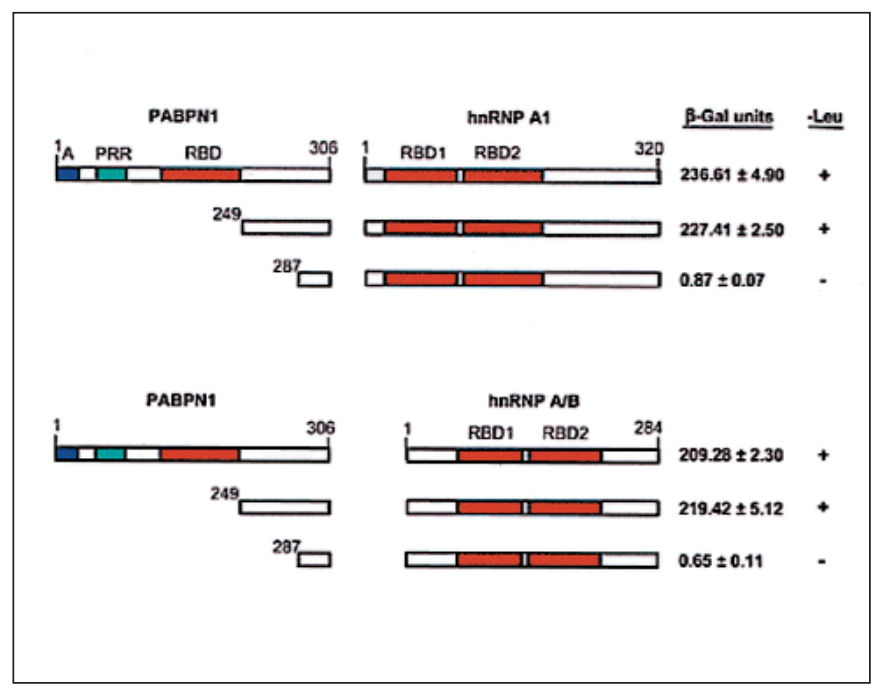

Figure 1: Yeast two-hybrid analysis of the interactions between PABPN1 and hnRNP A1 or A/B. AA249-306 of PABPN1 was used as bait to screen a human fetal brain cDNA library. The LacZ and LEU2 were used as reporter genes. The interaction between two co-expressed proteins was characterized as positive if the yeast cells express $\beta$ galactosidase (LacZ gene activated) and grew in the absence of leucine (LEU2 gene activated). $\beta$-galactosidase activity was measured using $\beta$ gal liquid assay. In the -leucine column, (+): yeast cells grow in the absence of leucine; (-): not grow. A: alanine stretch; PRR: proline rich region; $R B D$ : putative $R N A$ binding domain.
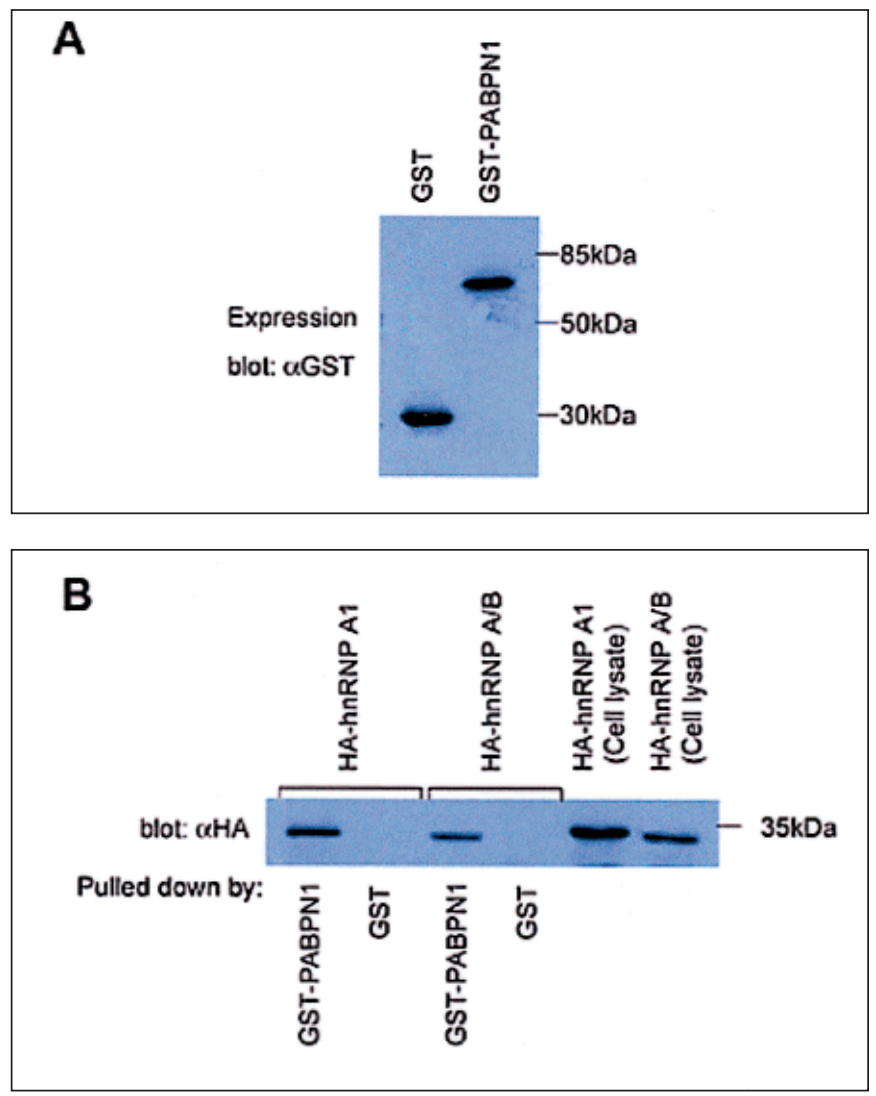

Figure 2: GST pull-down analysis of the interaction between PABPN1 and hnRNP A1 or A/B. (A) Expression of GST and GST-PABPN1. The proteins were expressed in bacteria, purified using Bulk and RediPack GST Purification Modules, and detected using anti-GST polyclonal antibody. (B) The hnRNP $A 1$ and $A / B$ were $H A$ tagged, expressed in yeast cells and confirmed by Western blotting using anti-HA polyclonal antibody (the right two lanes). Yeast cells were lysed and the lysate was incubated with $5 \mu \mathrm{g}$ purified GST or GST-PABPN1 obtained from A, pulled-down by Glutathione Sepharose $4 B$ beads, and assayed by Western blotting using the HA antibody.

\section{Immunoprecipitation}

In 48 hours post-transfection, the COS-7 cells co-expressing GFP-wtPABPN1 and HA-hnRNP A1 or A/B were washed twice with ice-cold phosphate buffer, lysed using Nonidet-P-40 lysis buffer $(50 \mathrm{mM}$ Tris pH 7.5, $150 \mathrm{mM} \mathrm{NaCl}, 10 \%$ glycerol, $0.5 \%$ NP-40, protease inhibitors) and further disrupted by homogenizing. The lysate was briefly spined. The supernatant was added with DNase 1 and RNase A to remove DNA and RNA, and incubated overnight at $4^{\circ} \mathrm{C}$ with $5 \mu \mathrm{g}$ of polyclonal GFP antibody (Clontech, Palo Alto, CA) and $50 \mu$ l of Protein A Sepharose 4 Fast Flow suspension (Amersham Pharmacia Biotech, Piscataway, NJ). The beads were washed six times with washing buffer $(50 \mathrm{mM}$ Tris $\mathrm{pH} 7.5,150 \mathrm{mM} \mathrm{NaCl}, 10 \%$ glycerol, $0.1 \% \mathrm{NP}-40$ ) and suspended in $40 \mu \mathrm{l}$ of protein sample buffer and heated to $95^{\circ} \mathrm{C}$ for five minutes. The immunoprecipitates were resolved on $12 \%$ SDS-PAGE, probed with antiHA antibody. The same membrane was stripped and re-probed with anti-GFP antibody. 

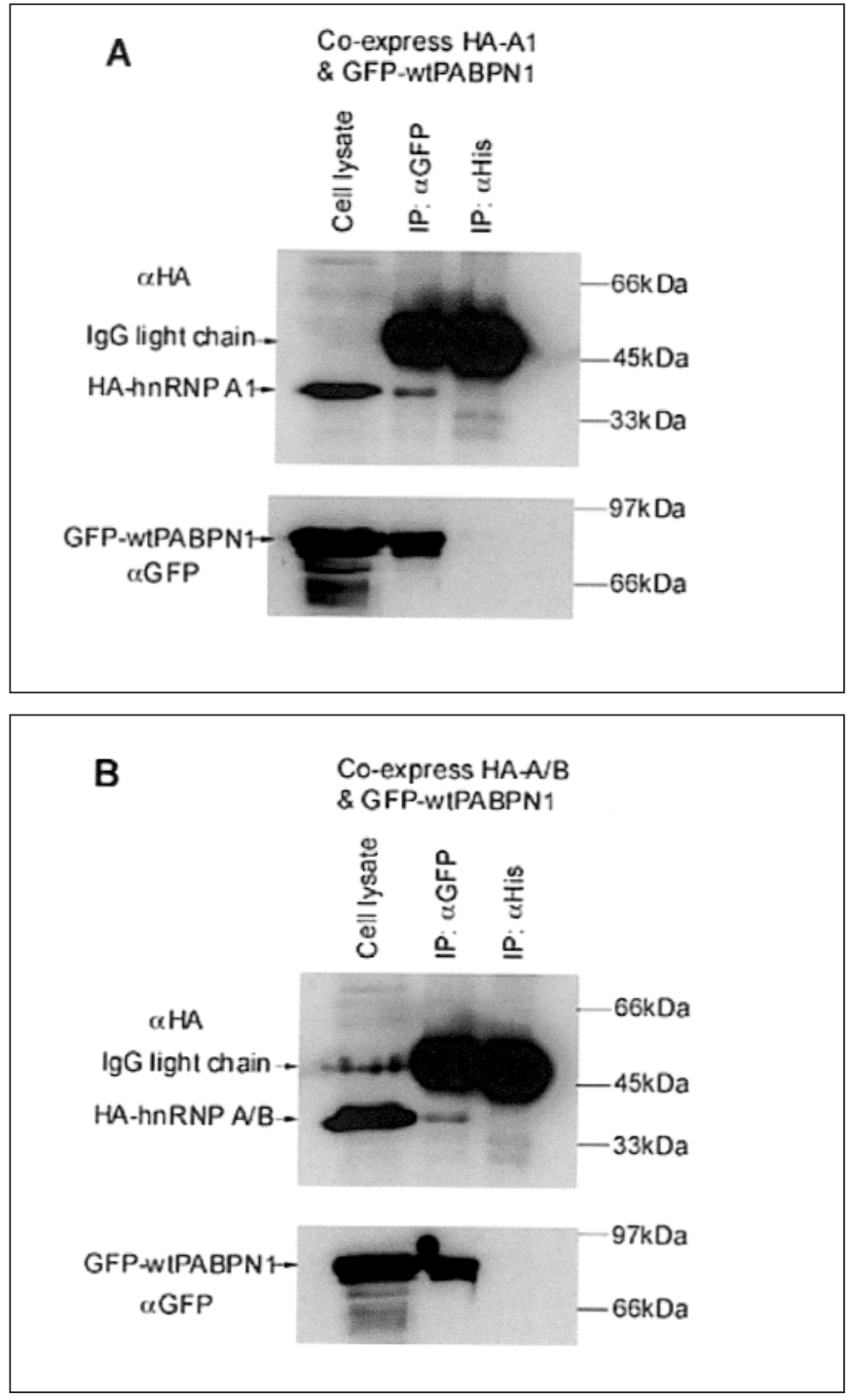

Figure 3: Co-immunoprecipitaion of hnRNP $A 1$ and $A / B$ with wtPABPN1. (A) GFP-wtPABPN1 and HA-hnRNP Al were co-expressed in COS-7 cells. The GFP-wtPABPN1 was immunoprecipitated using polyclonal GFP antibody and the immunoprecipitates were resolved on SDS-PAGE and blotted using anti-HA polyclonal antibody to check the presence of HA-hnRNP Al (middle lane). The polyclonal anti-Histidine antibody was used as a negative control (right lane). The cell lysate without immunoprecipitation was used to check the expression of HAhnRNP Al (left lane). The same Western blot was stripped and redetected with anti-GFP antibody to confirm the presence of GFPwtPABPN1 in the immunoprecipitates (lower panel). (B) The same experiment as $(A)$ was performed except using hnRNP A/B instead of $A 1$.

\section{RESULTS}

\section{hnRNPA1 and A/B interact with PABPN1}

The yeast two hybrid system was used to search for proteins interacting with the C-terminal domain of PABPN1 (AA249306) that excludes the putative RNA binding domain. $3 \times 10^{6}$ clones were screened, and 20 positive clones were isolated from a human fetal brain cDNA library, seven of which encoded hnRNP A1, and five encoded hnRNP A/B. The hnRNP A/B was previously named C-type hnRNP protein, ${ }^{44}$ and subsequently renamed to type A/B hnRNP protein. ${ }^{45}$ The full-length cDNA for hnRNP A1 and A/B were obtained from Integrated Molecular Analysis of the Human Genome and its Expression (I.M.A.G.E.) (ID A1: 549503; A/B: 610850), and the full-length hnRNP A1 and $\mathrm{A} / \mathrm{B}$ proteins were tested for interacting to PABPN1. Both full-length PABPN1 and its C-terminus strongly interact with hnRNP A1 or A/B, while the last 20 amino acid segment (AA287-306) does not interact with either hnRNP A1 or A/B (Figure 1).

\section{Interaction between PABPN1 and hnRNP A1 or A/B was confirmed by GST pull-down and immunoprecipitation assays}

To confirm the observed interaction, we performed a GST pull-down assay. PABPN1 was fused to GST, while hnRNP A1 and $\mathrm{A} / \mathrm{B}$ were $\mathrm{HA}$ tagged. The GST tagged proteins were incubated with HA tagged proteins and pulled down by glutathione sepharose 4B beads. The pulled-down proteins were resolved on SDS-PAGE, and blotted with anti-HA antibody. GST-PABPN1 is able to pull-down HA tagged hnRNP A1 and A/B while GST alone is not (Figure 2). In co-immunoprecipitation assay, the wild-type PABPN1 (wtPABPN1) was fused to the green fluorescent protein (GFP), while hnRNP A1 and A/B were HA-tagged. GFP and HA fusion proteins were coexpressed in COS-7 cells. The cells were homogenized and the lysates were treated with DNase 1 and RNase A to digest the DNA and RNA. The GFP fusion proteins were immunoprecipitated using GFP polyclonal antibody, and the immunoprecipitates were resolved on SDS-PAGE and blotted with anti-HA antibody. Both HA tagged hnRNP A1 and A/B are co-immunoprecipitated with GFP-wtPABPN1, but cannot be precipitated using polyclonal anti-histidine antibody (Figure 3).

\section{Polyalanine-expanded PABPN1 induces insoluble intranuclear aggregates}

It was reported that the expression of polyalanine-expanded PABPN1 (mPABPN1) in COS-7 cells induces large insoluble intranuclear protein aggregates. ${ }^{39-42}$ We expressed GFPwtPABPN1, GFP-mPABPN1 (with 17 alanines), HA-hnRNP A1 and HA-hnRNP A/B in COS-7 cells. Three days posttransfection, the transfected cells were either fixed or treated with $0.5 \mathrm{M} \mathrm{KCl}$ in HPEM before fixation. Potassium chloride treatment can remove soluble proteins that are not associated with insoluble pathological protein aggregates. ${ }^{36}$ The HA tagged proteins were immunodetected using the anti-HA antibody and a rhodamine conjugated secondary antibody. All the expressed proteins are predominantly localized to the nucleus (Figure 4). GFP-wtPABPN1, HA-hnRNP A1 and A/B are soluble proteins and are therefore not resistant to $\mathrm{KCl}$ treatment. However, the aggregates induced by mPABPN1 are resistant to $\mathrm{KCl}$ treatment.

hnRNP A1 and A/B co-localize with MPABPN1 to the intranuclear insoluble aggregates

The hnRNP proteins are predominantly nuclear RNA binding proteins and distribute throughout the nucleoplasm (Figure 4). In order to examine whether hnRNP A1 and A/B are involved in intranuclear protein aggregation induced by mPABPN1, the HA 


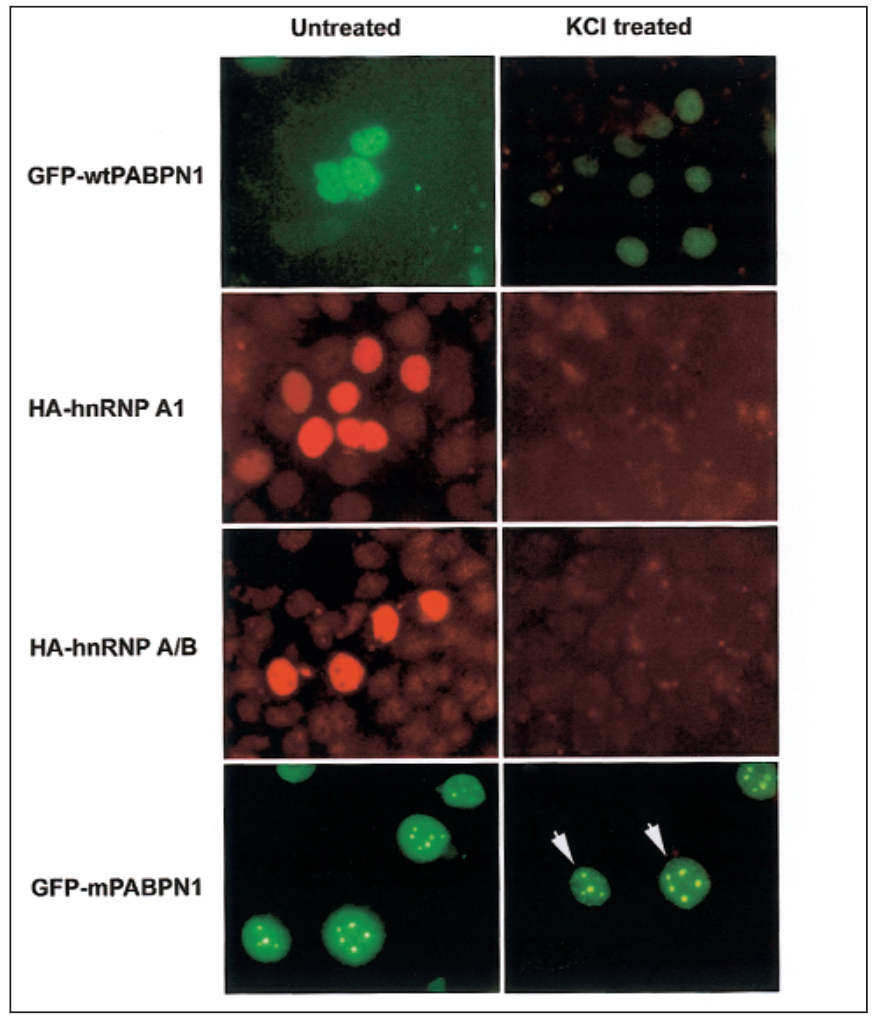

Figure 4: Expression of $M P A B P N 1$ induces insoluble intranuclear aggregates. GFP-wtPABPN1, HA-hnRNP Al, HA-hnRNP A/B, and GFP-mPABPN1 were transiently expressed in COS-7 cells. Three days post-transfection, the transfected cells were either fixed (left panels) or treated with $0.5 \mathrm{M} \mathrm{KCl}$ in HPEM buffer to remove the soluble proteins (right panel). The HA-hnRNPs expressed cells were immunostained using anti-HA antibody. Arrowheads indicate the nuclei containing insoluble aggregates induced by $M P A B P N 1$.

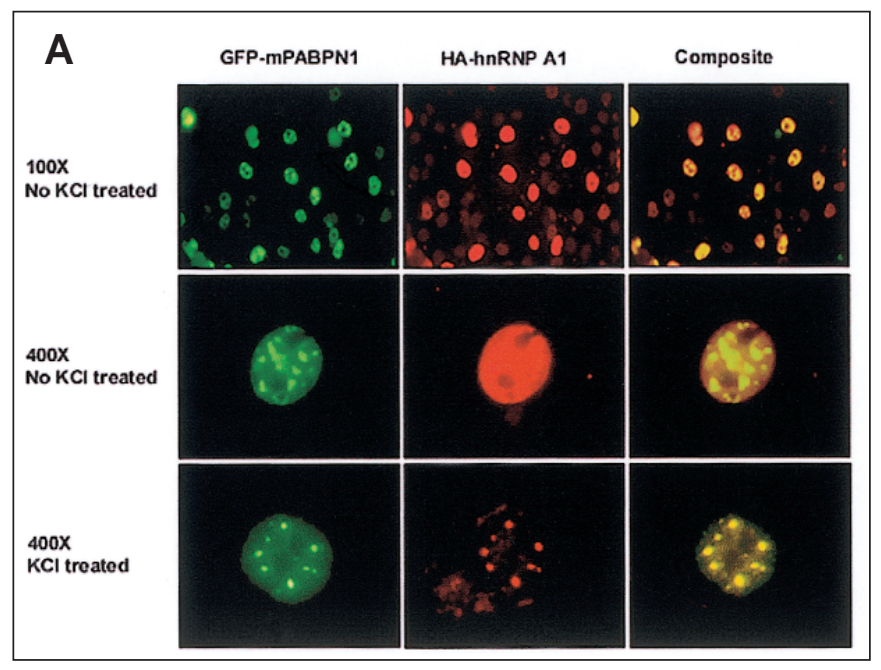

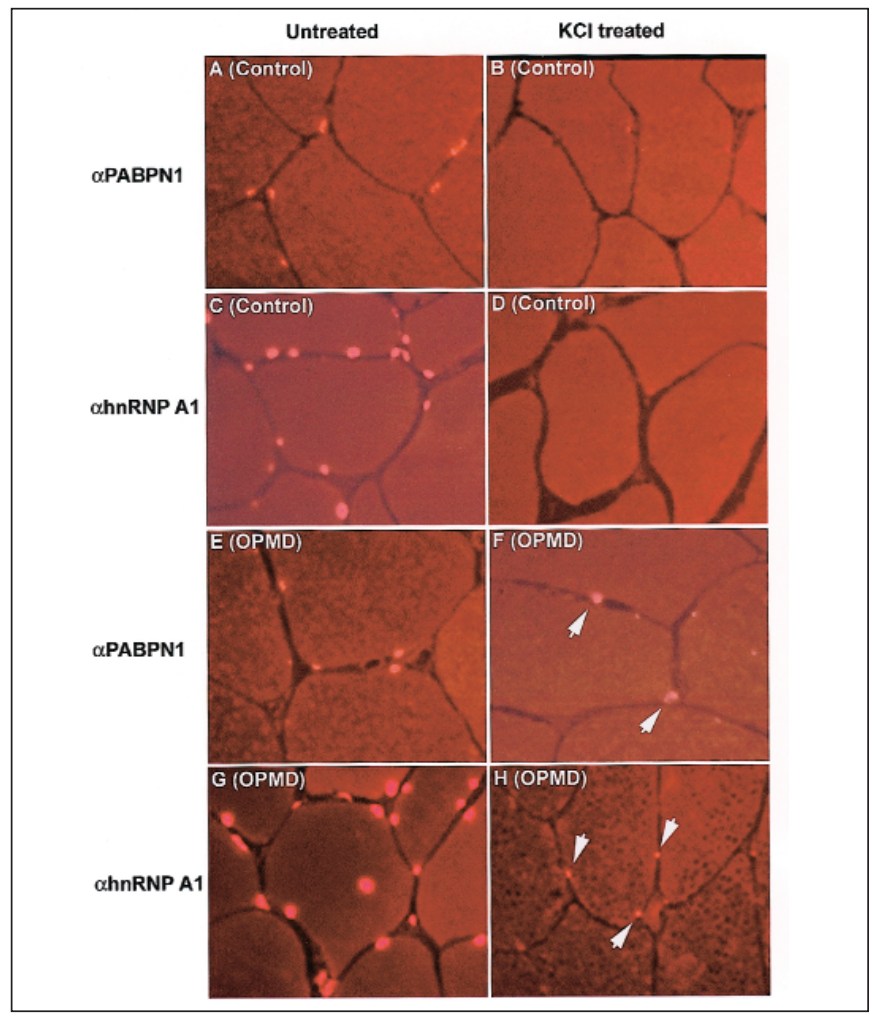

Figure 6: The intranuclear inclusions in OPMD muscle nuclei sequester $P A B P N 1$ and hnRNP A1. Immunohistochemistry was performed on cross microtome sections of the deltoid muscle from a control subject $(A, B, C$, and $D)$ and an OPMD patient $(E, F, G$ and $H)$. Sections were either immunostained without $K C l$ treatment $(A, C, E$, and $G)$ or treated with $1 \mathrm{M} \mathrm{KCl}$ in HPEM buffer for $5 \mathrm{~min}$. at room temperature before immunustaining to remove soluble proteins $(B, D, F$ and $H)$. Immunohistochemistry was done using polyclonal anti-PABPN1 antibody $(A, B, E$ and $F)$ or monoclonal anti-hnRNP Al antibody $(C, D$, $G$ and $H$ ), and rhodamine conjugated secondary antibody. Arrowheads indicate the positively stained insoluble intranuclear inclusions.

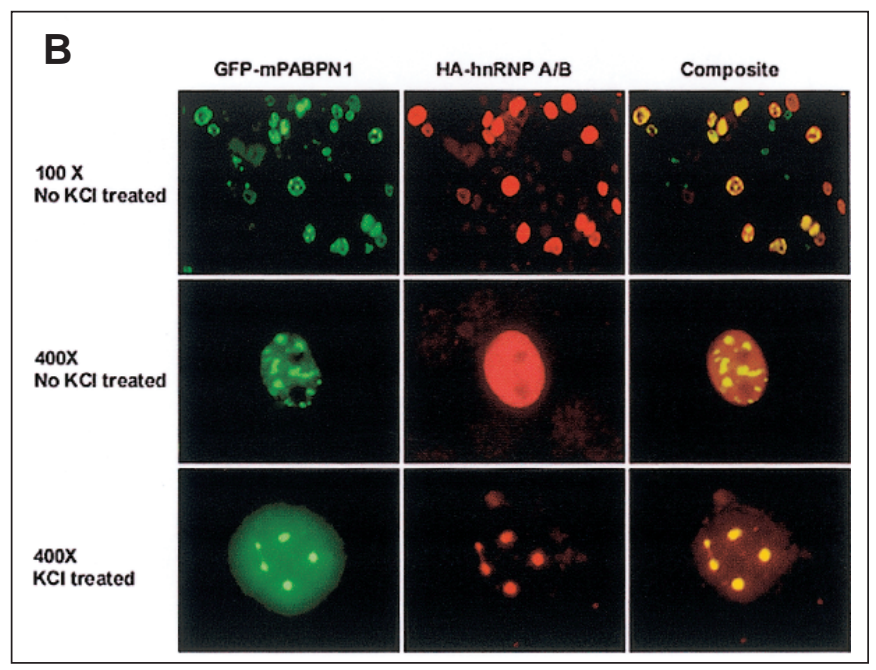

Figure 5: The hnRNP Al and A/B co-localize with $m P A B P N 1$ to the insoluble intranuclear aggregates. (A) HA-hnRNP Al and GFP-mPABPN1 were co-expressed in COS-7 cells. In 72 hrs, the cells were either fixed ( $a$ and b) or treated with 0.5M KCl in HPEM buffer before fixation (c). The HAhnRNP A1 was immunostained using an anti-HA polyclonal antibody and rhodamine conjugated secondary antibody (in red). Pictures were taken under low magnification to show the efficiency of transfection (a), high magnification to enable to see the aggregates ( $b$ and $c)$, and composition to show the co-localization (left). (B) The same experiment as (A) was performed except using hnRNP A/B instead of A1. 
tagged hnRNP proteins were co-expressed with GFP-mPABPN1, and detected by immunocytochemistry using anti-HA antibody and a rhodamine conjugated secondary antibody. Without $\mathrm{KCl}$ treatment, $\mathrm{A} 1$ and $\mathrm{A} / \mathrm{B}$ are predominantly localized to the nucleus (Figures 4 and 5). After $\mathrm{KCl}$ treatment, the soluble A1 and $\mathrm{A} / \mathrm{B}$ are removed, but the $\mathrm{KCl}$ resistant aggregates still contain hnRNP A1 and A/B signals (Figure 5). These signals colocalize completely with mPABPN1 aggregates (see the gold aggregates in Figure 5).

\section{Insoluble OPMD nuclear inclusions contain hnRNP A1}

PABPN1 in nuclear inclusions of the muscle from OPMD patients is insoluble and resistant to $\mathrm{KCl}$ treatment, whereas the protein localized in the nucleoplasm is solubilized. ${ }^{36,40}$ In order to confirm whether hnRNP A1 is part of the insoluble nuclear inclusions from the OPMD patient, we detected the presence of PABPN1 and hnRNPA1 in muscle nuclei from an OPMD patient and a control subject using immunohistochemistry (Figure 6). In the control subject, both PABPN1 and hnRNP A1 are soluble and not resistant to $\mathrm{KCl}$ treatment (Figures 6B and 6D), whereas in the OPMD patient, both PABPN1 and hnRNP A1 localize to the insoluble nuclear inclusions that are resistant to $\mathrm{KCl}$ treatment (Figures $6 \mathrm{~F}$ and $6 \mathrm{H}$ ). We also performed co-immunostaining using polyclonal anti-PABPN1 and monoclonal anti-A1 antibodies. Unfortunately, these two antibodies were not compatible and failed to pick up PABPN1 and A1 on the same section. The presence of hnRNP A/B in OPMD nuclear inclusions could not be detected due to the lack of efficient antihnRNP A/B antibody.

\section{Discussion}

The yeast two-hybrid library screen is an efficient and sensitive method to look for protein interacting partners but, with RNA binding proteins, false positive results may occur since RNA can bridge two RNA binding proteins together. It was previously shown that a double point mutant of PABPN1 in the putative RNA binding domain ( $\mathrm{Y}$ to $\mathrm{A}$ at position $175, \mathrm{~F}$ to $\mathrm{A}$ at position 215) loses its RNA activity, ${ }^{46}$ indicating that the Cterminus AA249-306 does not have RNA binding activity. We therefore used the C-terminus of PABPN1 from AA249-306, excluding the putative RNA binding domain, as bait to screen the cDNA library, and identified PABPN1 interacting proteins hnRNP A1 and A/B. The observations were further confirmed by GST pull-down and immunoprecipitation assays, indicating the interactions between PABPN1 and these hnRNP proteins are real, and not false positives caused by their RNA binding activities. PABPN1 coats the RNA poly(A) tail, while hnRNP proteins bind to the rest of the RNA molecule. The binding domain in PABPN1 for hnRNP A1 or A/B is located in the Cterminus that does not overlap the putative RNA binding domain, suggesting that PABPN1 is able to interact with these hnRNP proteins while binding to the poly(A) tail. We have recently found that the last 18 residues of PABPN1 from AA289-306 serve as a nuclear localization signal (NLS) for this protein (unpublished observation). The segment of PABPN1 from AA287-306 does not interact with hnRNP A1 and A/B, implying that the NLS of PABPN1 is not the binding domain for these two hnRNP proteins.

PABPN1 is an abundant nuclear protein that normally forms nuclear speckles, ${ }^{47}$ while expression of mPABPN1 with expanded polyalanines in cells induces the formation of large intranuclear aggregates. ${ }^{39-42}$ hnRNP A1 and A/B are also predominantly localized to the nucleus, but do not form speckles (Figure 4). PABPN1 present in the OPMD inclusions is resistant to $\mathrm{KCl}$ treatment, like other pathological aggregates that are typically insoluble in $\mathrm{KCl} .{ }^{36}$ The wtPABPN1, hnRNP A1 or A/B expressed in COS-7 cells are not resistant to $\mathrm{KCl}$ treatment, while the large intranuclear aggregates induced by mPABPN1 are. These observations suggest that intranuclear aggregates induced by $\mathrm{mPABPN} 1$ have the same insoluble property as the ones found in OPMD patients. When hnRNP A1 and A/B are coexpressed with mPABPN1 in COS-7 cells, the hnRNP proteins co-localize to the aggregates that are resistant to $\mathrm{KCl}$ treatment. Studies on muscle tissue from OPMD patients also showed that hnRNP A1 is localized to the insoluble OPMD nuclear inclusions. Thus, we conclude that OPMD intranuclear inclusions sequester hnRNP proteins that interact with PABPN1.

Oculopharyngeal muscular dystrophy is caused by the expansion of a polyalanine stretch in PABPN1 from 10 to 12-17 residues, ${ }^{12}$ which leads to intranuclear protein aggregation and cellular toxicity. ${ }^{39-42}$ In addition to genetic evidence, PABPN1 is detected in OPMD nuclear inclusions using immunohistochemistry, ${ }^{36,37}$ suggesting a direct role in protein aggregation. We have recently found two oligomerization domains that are located in the C-terminus of PABPN1 far from the polyalanine stretch. Inactivating oligomerization by deletions in either of the oligomerization domains prevents intranuclear protein aggregation and reduces death rate of the cells expressing the non-oligomerizing mPABPN $1 .{ }^{40}$ Those observations suggest that OPMD intranuclear aggregates might be toxic and critical in initiating OPMD pathogenesis. That raises the question of why OPMD intranuclear aggregates are toxic. Patient studies showed that OPMD nuclear inclusions sequester poly(A) RNA, suggesting that these inclusions might be "mRNA traps" that interfere with mRNA export. ${ }^{36}$ Using the N-terminus of PABPN1 as bait in yeast two hybrid screen, Kim et al ${ }^{41}$ recently identified a PABPN1 interaction with SKIP, a potential co-transcription factor working with PABPN1 in muscle cells to stimulate muscle-specific gene expressions. SKIP might be sequestered in the OPMD intranuclear inclusions. Identification of hnRNP proteins in OPMD intranuclear inclusions suggests that: 1) OPMD intranuclear inclusions sequester PABPN1 interacting proteins; 2) OPMD intranuclear inclusions interfere with mRNA export as hnRNP proteins are involved in this function.

The hnRNP proteins are predominantly nuclear RNA-binding proteins associated specifically with pre-mRNA and mRNA molecules. ${ }^{48,49}$ The nucleocytoplasmic shuttling protein, hnRNP A1 has been reported to play a direct role in mRNA export. ${ }^{49-52}$ PABPN1 is also a shuttling protein, coating the poly(A) tail of mRNA in the nucleus, and is suggested to involve in mRNA export. ${ }^{46}$ hnRNP A1 and A/B were identified using the Cterminus of PABPN1, suggesting that the hnRNPs can interact with the wild-type PABPN1. The interaction between hnRNP A1 and PABPN1 is probably required for packaging mRNA for export. Identifying hnRNP A1 in OPMD intranuclear aggregates provides evidence that OPMD aggregates may interfere with mRNA export.

The hnRNP A1 protein is also involved in pre-mRNA 
splicing, telomere length maintenance, transcription regulation and pre-mRNA 3 '-end processing. ${ }^{53}$ Sequestering some hnRNP A1 molecules in the OPMD aggregates probably reduce the normal concentration of this protein in the nucleus and affect its functions. It could also be detrimental to the cell if, to carry out its normal function, hnRNP A1 needs its interacting partner PABPN1 which is largely aggregated in the OPMD inclusions. Therefore, it is possible that the intranuclear inclusion might interfere with hnRNP functions.

The hnRNP A/B is homologous to hnRNP A1.45,48 The binding of hnRNP A/B to RNA disrupts the residual secondary structure of RNAs. ${ }^{48}$ Its isoform protein was cloned in 1997 and named ABBP-1. ${ }^{54}$ ABBP-1 binds apolipoprotein B (apoB) mRNA and is involved in apoB pre-mRNA editing. ${ }^{54} \mathrm{ApoB}$ mRNA editing is a post-transcriptional regulation, consists of a $\mathrm{C}$ to $\mathrm{U}$ conversion of the codon CAA, encoding glutamine 2153, to UAA, an in-frame stop codon in apoB mRNA. ${ }^{55}$ The process requires the presence of the $\operatorname{poly}(\mathrm{A})$ tail of apoB mRNA. ${ }^{56}$ Because the poly(A) tail of mRNA is always coated by PABPN1 molecules in the nucleus, the interaction between ABBP-1 and PABPN1 implies that PABPN1 might be involved in apoB mRNA editing. Sequestering hnRNP A/B protein in OPMD intranuclear inclusions suggests that the inclusions might interfere with mRNA processing and trap pre-mRNA.

The presence of abnormal protein aggregates is a common finding in a number of neuronal degenerative diseases. ${ }^{18,57}$ Expansions of the polyglutamine stretch in the disease gene products lead to protein aggregation and abnormal association with various cell proteins. ${ }^{57,58}$ Pathogenesis studies showed that, although polyglutamine tracts themselves are very toxic, residues outside of the polyglutamine tract in each diseasecausing protein have an important role in defining the course and specificity of disease. ${ }^{58}$ These residues participate in important cellular processes such as the subcellular localization of the polyglutamine protein and its interaction with other cellular molecules important in disease progression. ${ }^{58}$ These observations also apply to OPMD, since OPMD aggregates sequester PABPN1 interacting proteins such as hnRNPs, SKIP, ${ }^{41}$ and mRNAs. ${ }^{36}$ Interestingly there is no evidence indicating that polyalanine expanded PABPN1 loses any of its known functions. In vitro polyadenylation assay showed that polyalanine expanded PABPN1 is still able to stimulate RNA poly(A) tail synthesis and control its length (unpublished observation). The poly(A) length of mRNA isolated from muscle of OPMD patients is not affected. ${ }^{36}$ However, polyalanine expanded PABPN1 has a gainof-function that induces the formation of large intranuclear protein aggregates, ${ }^{39-42}$ and these aggregates are toxic and associated with cell death. ${ }^{40}$ We suggest that OPMD intranuclear inclusions that trap mRNA and sequester PABPN1 interacting proteins play critical roles on this disease progression.

\section{ACKNOWLEDGEMENTS}

We thank Dr. G. Dreyfuss for providing monoclonal antibody against hnRNP A1; Drs. P. Lau and L. Chen for antibody against hnRNP A/B and discussing with us about the roles of ABBP-1 and poly(A) tail in apoB pre-RNA editing; to Dr. M. Jannatipour for showing us the yeast two-hybrid screen techniques; Mr. D. Rochefort for his invaluable suggestions on construct making. We thank Drs. C. Gaspar, and A. Toulouse for careful reading and comments on the manuscript.

This work was supported by the Muscular Dystrophy Association
(USA) and the Federation Foundation of Greater Philadelphia. B.B. is a chercheur-boursier of the FRSQ. G.A.R. is supported by the CIHR.

\section{REFERENCES}

1. Brais B, Rouleau GA, Bouchard JP, Fardeau M, Tome FM. Oculopharyngeal muscular dystrophy. Semin Neurol 1999; 19: 59-66.

2. Muller T, Schroder R, Zierz S. GCG repeats and phenotype in oculopharyngeal muscular dystrophy. Muscle Nerve 2001; 24: 120-122.

3. Blumen SC, Korczyn AD, Lavoie H, et al. Oculopharyngeal MD among Bukhara Jews is due to a founder (GCG)9 mutation in the PABP2 gene. Neurology 2000; 55: 1267-1270.

4. Nagashima T, Kato H, Kase M, et al. Oculopharyngeal muscular dystrophy in a Japanese family with a short GCG expansion (GCG)(11) in PABP2 gene. Neuromuscul Disord 2000; 10: 173177.

5. Mirabella M, Silvestri G, Di Giovanni S, et al. GCG genetic expansions in Italian patients with oculopharyngeal muscular dystrophy. Neurology 2000; 54: 608-614.

6. Grewal RP, Karkera JD, Grewal RK, Detera-Wadleigh SD. Mutation analysis of oculopharyngeal muscular dystrophy in Hispanic American families. Arch Neurol 1999; 56: 1378-1381.

7. Tome FM, Fardeau M. Nuclear inclusions in oculopharyngeal dystrophy. Acta Neuropathol (Berl) 1980; 49: 85-87.

8. Tome FM, Chateau D, Helbling-Leclerc A, Fardeau M. Morphological changes in muscle fibers in oculopharyngeal muscular dystrophy. Neuromuscul Disord 1997; 7 (Suppl 1): S63-69.

9. Brais B, Xie YG, Sanson M, et al. The oculopharyngeal muscular dystrophy locus maps to the region of the cardiac alpha and beta myosin heavy chain genes on chromosome 14q11.2-q13. Hum Mol Genet 1995; 4: 429-434.

10. Brais B, Bouchard JP, Gosselin F, et al. Using the full power of linkage analysis in 11 French Canadian families to fine map the oculopharyngeal muscular dystrophy gene. Neuromuscul Disord 1997; 7 (Suppl 1): S70-74.

11. Xie YG, Rochefort D, Brais B, et al. Restriction map of a YAC and cosmid contig encompassing the oculopharyngeal muscular dystrophy candidate region on chromosome 14q11.2-q13. Genomics 1998; 52: 201-204.

12. Brais B, Bouchard JP, Xie YG, et al. Short GCG expansions in the PABP2 gene cause oculopharyngeal muscular dystrophy. Nat Genet 1998; 18: 164-167.

13. Muragaki Y, Mundlos S, Upton J, Olsen BR. Altered growth and branching patterns in synpolydactyly caused by mutations in HOXD13. Science 1996; 272: 548-551.

14. Mundlos S, Otto F, Mundlos C, et al. Mutations involving the transcription factor CBFA1 cause cleidocranial dysplasia. Cell 1997; 89: 773-779.

15. Brown SA, Warburton D, Brown LY, et al. Holoprosencephaly due to mutations in ZIC2, a homologue of Drosophila odd-paired. Nat Genet 1998; 20: 180-183.

16. Goodman FR, Bacchelli C, Brady AF, et al. Novel HOXA13 mutations and the phenotypic spectrum of hand-foot-genital syndrome. Am J Hum Genet 2000; 67: 197-202.

17. Crisponi L, Deiana M, Loi A, et al. The putative forkhead transcription factor FOXL2 is mutated in blepharophimosis/ ptosis/epicanthus inversus syndrome. Nat Genet 2001; 27: 159166.

18. Ferrigno P, Silver PA. Polyglutamine expansions: proteolysis, chaperones, and the dangers of promiscuity. Neuron 2000; 26: 912.

19. Huntington's Disease Collaborative Research Group. A novel gene containing a trinucleotide repeat that is expanded and unstable on Huntington's disease chromosomes. Cell 1993; 72: 971-983.

20. La Spada AR, Wilson EM, Lubahn DB, Harding AE, Fischbeck KH. Androgen receptor gene mutations in X-linked spinal and bulbar muscular atrophy. Nature 1991; 352: 77-79.

21. Koide R, Ikeuchi T, Onodera O, et al. Unstable expansion of CAG repeat in hereditary dentatorubral-pallidoluysian atrophy 
(DRPLA). Nat Genet 1994; 6: 9-13.

22. Nagafuchi S, Yanagisawa $H$, Sato $K$, et al. Dentatorubral and pallidoluysian atrophy expansion of an unstable CAG trinucleotide on chromosome 12p. Nat Genet 1994; 6: 14-18.

23. Orr HT, Chung MY, Banfi S, et al. Expansion of an unstable trinucleotide CAG repeat in spinocerebellar ataxia type 1 . Nat Genet 1993; 4: 221-226.

24. Kawaguchi Y, Okamoto T, Taniwaki M, et al. CAG expansions in a novel gene for Machado-Joseph disease at chromosome 14q32.1. Nat Genet 1994; 8: 221-228.

25. Imbert G, Saudou F, Yvert G, et al. Cloning of the gene for spinocerebellar ataxia 2 reveals a locus with high sensitivity to expanded CAG/glutamine repeats. Nat Genet 1996; 14: 285-291.

26. Pulst SM, Nechiporuk T, Gispert S, et al. Moderate expansion of a normally biallelic trinucleotide repeat in spinocerebellar ataxia type 2. Nat Genet 1996; 14: 269-276.

27. Sanpei K, Takano H, Igarashi S, et al. Identification of the spinocerebellar ataxia type 2 gene using a direct identification of repeat expansion and cloning technique, DIRECT. Nat Genet 1996; 14: 277-284.

28. David G, Abbas N, Stevanin G, et al. Cloning of the SCA7 gene reveals a highly unstable CAG repeat expansion. Nat Genet 1997; 17: 65-70.

29. Koob MD, Benzow KA, Bird TD, et al. Rapid cloning of expanded trinucleotide repeat sequences from genomic DNA. Nat Genet 1998; 18: 72-75.

30. Wahle E. A novel poly(A)-binding protein acts as a specificity factor in the second phase of messenger RNA polyadenylation. Cell 1991; 66: 759-768.

31. Colgan DF, Manley JL. Mechanism and regulation of mRNA polyadenylation. Genes Dev 1997; 11: 2755-2766.

32. Minvielle-Sebastia L, Keller W. mRNA polyadenylation and its coupling to other RNA processing reactions and to transcription. Curr Opin Cell Biol 1999; 11: 352-357.

33. Barabino SML, Keller W. Last but not least: regulated poly(A) tail formation. Cell 1999; 99: 9-11

34. Wahle E. Poly(A) tail length control is caused by termination of processive synthesis. J Biol Chem 1995; 270: 2800-2808.

35. Bienroth S, Keller W, Wahle E. Assembly of a processive messenger RNA polyadenylation complex. EMBO J 1993; 12: 585-594.

36. Calado A, Tome FMS, Brais B, et al. Nuclear inclusions in oculopharyngeal muscular dystrophy consist of poly(A) binding protein 2 aggregates which sequester poly(A) RNA. Hum Mol Genet 2000; 9: 2321-2328.

37. Becher MW, Kotzuk JA, Davis LE, Bear DG. Intranuclear inclusions in oculopharyngeal muscular dystrophy contain poly(A) binding protein 2. Ann Neurol 2000; 48: 812-815.

38. Uyama E, Tsukahara T, Goto K, et al. Nuclear accumulation of expanded PABP2 gene product in oculopharyngeal muscular dystrophy. Muscle Nerve 2000; 23: 1549-1554.

39. Shanmugam V, Dion P, Rochefort D, et al. PABP2 polyalanine tract expansion causes intranuclear inclusions in oculopharyngeal muscular dystrophy. Ann Neurol 2000; 48: 798-802.

40. Fan X, Dion P, Laganiere J, Brais B, Rouleau GA. Oligomerization of polyalanine expanded PABPN1 facilitates nuclear protein aggregation that is associated with cell death. Hum Mol Genet 2001; 10: 2341-2351.

41. Kim YJ, Noguchi S, Hayashi YK, et al. The product of an oculopharyngeal muscular dystrophy gene, poly(A)-binding protein 2, interacts with SKIP and stimulates muscle-specific gene expression. Hum Mol Genet 2001; 10: 1129-1139.

42. Bao YP, Cook LJ, O'Donovan D, et al. Mammalian, yeast, bacterial and chemical chaperones reduce aggregate formation and death in a cell model of oculopharyngeal muscular dystrophy. J Biol Chem 2002; 277:12263-12269.

43. Nakielny S, Dreyfuss G. Transport of proteins and RNAs in and out of the nucleus. Cell 1999; 99: 677-690.

44. Kumar A, Sierakowska H, Szer W. Purification and RNA binding properties of a C-type hnRNP protein from HeLa cells. J Biol Chem 1987; 262: 17126-17137.

45. Khan FA, Jaiswal AK, Szer W. Cloning and sequence analysis of a human type A/B hnRNP protein. FEBS 1991; 290: 159-161.

46. Calado A, Kutay U, Kuhn U, Wahle E, Carmo-Fonseca M. Deciphering the cellular pathway for transport of poly(A)binding protein II. RNA 2000; 6: 245-256.

47. Krause S, Fakan S, Weis K, Wahle E. Immunodetection of poly(A) binding protein II in the cell nucleus. Exp Cell Res 1994; 214: 7582

48. Dreyfuss G, Matunis MJ, Pino-Roma S, Burd CG. hnRNP proteins and the biogenesis of mRNA. Ann Rev Biochem 1993; 62: 289321.

49. Swanson MS. Functions of nuclear pre-mRNA/mRNA binding proteins. In: Lamond AI, (Ed). Pre-mRNA Processing. Berlin/ New York: Springer Verlag, 1995: 17-33.

50. Pinol-Roma S, Dreyfuss G. Shuttling of pre-mRNA binding proteins between nucleus and cytoplasm. Nature 1992; 355: 730732.

51. Visa N, Alzhanova-Ericsson AT, Sun X, Kiseleva E. A pre-mRNAbinding protein accompanies the RNA from the gene through the nuclear pores and into polysomes. Cell 1996; 84: 253-264.

52. Nakielny S, Dreyfuss G. Nuclear export of proteins and RNAs. Curr Opin Cell Biol 1997; 9: 420-429.

53. Krecic AM, Swanson MS. hnRNP complexes: composition, structure, and function. Curr Opin Cell Biol 1999; 11: 363-371.

54. Lau PP, Zhu HJ, Nakamuta M, Chan L. Cloning of an apobec-1binding protein that also interacts with apolipoprotein $\mathrm{B}$ mRNA and evidence for its involvement in RNA editing. J Biol Chem 1997; 272: 1452-1455.

55. Innerarity TL, Boren J, Yamanaka S, Olofsson SO. Biosynthesis of apolipoprotein B48-containing lipoproteins. J Biol Chem 1996; 271: 2353-2356

56. Lau PP, Xiong W, Zhu HJ, Chen SH, Chan L. Apolipoprotein B mRNA editing is an intranuclear event that occurs posttranscriptionally coincident with splicing and polyadenylation. J Biol Chem 1991; 266: 20550-20554.

57. Sherman MY, Goldberg AL. Cellular defenses against unfolded proteins: a cell biologist thinks about neurodegenerative diseases. Neuron 2001; 29: 15-32.

58. Orr HT. Beyond the Qs in the polyglutamine diseases. Genes Dev 2001; 15: 925-932. 